\title{
DES GROUPES DE PAROLE AVEC LES ADOLESCENTS: UNE PAROLE SUBJECTIVÉE DANS L'INSTITUTION SCOLAIRE ${ }^{\star}$ \\ Laurence Gavarini ${ }^{\star}$
}

\begin{abstract}
RÉSUMÉ
Cet article présente un fragment d'une recherche "Co-psy enfant" sur la construction identitaire chez l'adolescent dans les repères de genre et de générations (identité sexuée, relations intergénérationnelles) telles qu'elle se manifeste à l'école. Elle s'est déroulée dans un collège et un lycée, situés dans des zones urbaines considérées comme très défavorisées. Nous évoquerons un temps fort de notre approche clinique avec la constitution de 7 groupes de parole d'adolescents. En résorbant en partie le clivage existant entre le statut d'élève et le Sujet adolescent, ces groupes ont montré l'intérêt d'instituer un espace pour la parole adolescente. Le moteur de sa création a été la demande des jeunes euxmêmes, véritable force instituante d'ordinaire négligée dans l'institution scolaire. Nous évoquerons la structuration de cet espace clinique des groupes de parole, leur fonction active de contenant pour les individus et leur mise en pratique d'un lien social habituellement impossible.
\end{abstract}

Mots clé: adolescents; subjectivation; genre; groupes de parole; approches cliniques

\section{DISCUSSION GROUPS WITH TEENAGERS:}

\section{A SPEECH SUBJECTIVISED IN THE SCHOOL}

\begin{abstract}
In connection with "Copsy-enfant" (French National Agency for Research, 20062009), our recent research on the construction of gender and generational identity among teenagers, we set up seven discussion groups for middle and high school students in a working-class neighborhood of metropolitan Paris. The purpose of this article is to situate our research within the context of today's interrogations on adolescence. Such groups provided for the teenagers who requested them, a space within the school reducing the gap between their pupil's status and their teenager's being Subject. In this paper, we speak about the structuration of these groups whose orientation was psychoanalytic. We address the containing function and the social bonds that such groups provided for the teenagers.

Keywords: adolescents; subjectification; gender; discussion groups; clinical approaches

$\star$ Intervention colloque Niteroi 3-11-2008

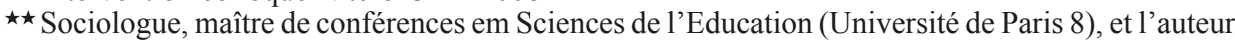
de La passion de l'enfant: filiation, procréation et éducation à l'aube du XXIème siècle. Paris: Denoël, 2001; (avec Françoise Petitot); La fabrique de l'enfant maltraité: un nouveau regard sur l'enfant et La famille. Ramonville Sainte-Agne: ERES, 1998.

E-mail:1gavarini@univ-paris8.fr
\end{abstract}


Nous venons de vivre une expérimentation à l'initiative des étudiants du département de psychologie de la UFF qu'ils avaient bien nommée Interférence et qui a accru mes sensations de déterritorialisation! En tous cas, cette expérimentation qui a supposé un certain assujettissement sonore et linguistique des participants, me semble pouvoir être mise en lien avec ce que je pensais vous dire aujourd'hui à propos des groupes de parole avec des adolescents dans l'institution scolaire. Dans un cas, les étudiants ont créé un dispositif ayant subverti l'ordre du colloque et la programmation de notre intervention. Dans l'autre, nous avons, nous-mêmes, construit un lieu de la parole dans l'institution scolaire qui a permis l'émergence d'une parole de sujets adolescents d'ordinaire aliénés dans l'exercice même de la parole, l'émergence d'une parole qui ne serait en tous cas pas que support d'une bonne communication. Alors je me suis demandé si au fond nous pouvions nous entendre, par delà les clivages portant notamment sur la référence à la psychanalyse, sur cette question de l'aliénation de la parole.

Merci donc de m'avoir donné cette occasion de parler avec vous de ma recherche dans le cadre de votre colloque! Il s'agit d'une recherche pluridisciplinaire entreprise depuis deux ans et qui porte sur les adolescents et leur construction identitaire dans les repères de genre et de générations, au regard des mutations sociales et subjectives actuelles. ${ }^{1}$ Elle s'est déroulée pour la partie que je coordonnais dans la proche banlieue de Paris. Un point fort de notre démarche a été constitué par l'animation de groupes de parole d'adolescents dans des établissements scolaires, un collège et un lycée, situés dans des zones urbaines considérées, en France, comme très défavorisées. Ce dispositif était complété par d'autres procédures d'investigation plus classiques: observations en situation scolaire et dans des moments informels, entretiens approfondis, observations de séquences de classes. En faisant varier plusieurs niveaux d'investigation, nous avons pu mesurer la diversité des registres situationnels, sociaux, groupaux et subjectifs convoqués par l'expérience scolaire des adolescents. En référence à Guattari, on pourrait ici parler de la transversalité de l'expérience adolescente, au plan subjectif et social, une transversalité souvent ignorée par ceux qui, professionnellement, ont à enseigner ou à prendre en charge des sujets adolescents.

Nous avons constaté une forte discontinuité entre l'élève à qui s'adresse l'école et les adolescents qu'ils sont, en fait, lorsqu'ils sont touchés dans leur intimité de sujets sexués et sexuels, lorsqu'ils sont interpellés dans leurs problématiques personnelles. Comme si, alors, les plans cognitifs et subjectifs se clivaient irrémédiablement, laissant ces sujets désemparés face au savoir, terrain où se manifeste prioritairement cet embarras. Ce clivage pourrait en évoquer d'autres qui se jouent dans toutes les institutions, aux articulations de l'individuel, de l'institutionnel et du politique, mais aussi aux articulations des logiques d'assujettissement à des fonctions dans l'institution (être élève, par exemple) et des logiques de subjectivation de l'expérience par les individus (être un adolescent en train de se construire). Sur la scène scolaire, c'est le rapport au savoir qui est l'arbitre de ce hiatus qu'éprouvent, à l'évidence plus que les autres, les jeunes issus des milieux 
populaires. Du point de vue de la norme scolaire, de la forme scolaire instituée, autant que du point de vue de leur inscription sociale, ils sont déjà des individus "par défaut" (CASTEL; HAROCHE, 2001), tels que Teresa Carreteiro en a, elle aussi, fait mention dans son intervention. L'opération scolaire rencontre en effet "l'opération adolescente", ainsi définie par des psychanalystes français, et participe sans doute de cette partition entre "individus par excès", répondant aux idéaux actuels de compétition et de promotion de soi dès le plus jeune âge, et individus "par défaut" ou "négatifs", parce qu'ils échappent précisément à cette course. En proposant des groupes de parole, nous ne prétendions pas résorber définitivement cette disparité mais en tous cas permettre que les adolescents puissent déposer une parole plus en phase avec eux-mêmes. L'enjeu était donc que cette parole soit entendue pour ce qu'elle disait et non, comme d'ordinaire, qu'elle soit mesurée à travers le prisme des normes scolaires et langagières.

Je vais donc évoquer les groupes de parole que nous avons animés dans des collèges et lycées en tant que dispositifs. A défaut de faire disparaître totalement ce clivage, ce hiatus entre l'élève et le sujet adolescent, ces groupes ont montré l'intérêt d'instituer un espace pour la parole du point de vue de la vie des sujets dans l'institution, mais aussi du strict point de vue de notre recherche. Nous nous sommes appuyés sur la demande des équipes et des jeunes eux-mêmes comme moteurs de la création de cette espace, utilisant ici une force instituante d'ordinaire négligée dans l'institution scolaire. C'est de la structuration de cet espace clinique des groupes de parole, de leur fonction active de contenant et de leur mise en pratique d'un lien social intériorisé (ROUCHY, 2006) que je souhaite parler ici.

\section{LA PAROLE DANS L'INSTITUTION}

Ma conception actuelle des groupes de parole présente quelques parentés avec une expérience clinique déjà ancienne (analyse institutionnelle) si on la considère sous l'angle des processus et dispositifs dynamiques que nous avons développés au sein de l'institution à partir d'une demande des sujets. Ces groupes prennent en partie leur source et inspiration dans la clinique institutionnelle tout en étant fort différents dans leur cadre et leur fonction. Ils viennent appuyer et soutenir ce qu'il convient de nommer forces instituantes (LOURAU, 1969, 1970; CASTORIADIS, 1975), sorte d'énergie collective capable de transformer les individus dans leurs contextes de travail, à la manière de ce que constitue la libido pour la psyché dans la cure analytique.

L'analyse institutionnelle reposait elle aussi, comme les groupes de parole que j'ai développés, sur un dispositif de parole, mais pensé sur le modèle des assemblées générales produites lors des mouvements sociaux: tous les acteurs directs, les ressortissants des différentes composantes de l'établissement étaient convoqués à ces assemblées. Les analystes travaillaient à cette ouverture vers une parole collective. La source métaphorique et mythique en était aussi l'Agora grecque, lieu de rencontre et de discussion, l'assemblée faisait de tous les mem- 
bres de l'institution rassemblés un sujet "politique" collectif. L'analyse relevait alors de la compréhension de l'institutionnalisation du dispositif et portait sur les effets de miroir et les écarts du dispositif assemblée générale par rapport à l'ordre institué surgissant à travers les analyseurs de cette situation créée. Par analogie, on pourrait dire que l'analyse institutionnelle entreprenait, sur la scène institutionnelle et groupale, une opération équivalente à celle qui se produit sur la scène de l'Inconscient pour la psychanalyse avec le transfert, les répétitions, les résistances, mais aussi les acting out et les passages à l'acte. Comme la psychosociologie dont elle a été proche, l'analyse institutionnelle travaillait aussi à partir des groupes et du lien social dans les institutions, tout en visant, par son action, des changements chez les individus. Une hypothèse, partagée avec la psychothérapie et la pédagogie institutionnelles, était qu'en analysant l'institution, les forces négatives qui la "travaillent", en prenant conscience des déterminants de leur place, en déliant leurs implications aux divers niveaux de leur inscription personnelle dans l'institution, les individus pourraient libérer une sorte d'énergie créatrice et s'émanciper de leurs emprises et assujettissements imaginaires. Les institutions pouvaient ainsi devenir moins anomiques, plus "thérapeutiques" et éducatives. J'ai retenu de cette clinique l'accent qu'elle mettait sur la nécessité de créer des espaces de discussion ouverts et nouveaux par rapport aux modes d'échanges institués souvent sclérosés, même si considérés de longue date comme des lieux de l'expression démocratique dans les institutions.

La manière dont j'ai investi les groupes de parole, renvoie à un idéal à l'origine de ma professionnalisation de jeune sociologue : celui d'occuper une fonction d'intervention en lien avec le changement social et individuel. Mais il est venu s'y greffer une disponibilité nouvelle aux Sujets en souffrance, étayée sur mon expérience de la psychanalyse, puis sur ma formation analytique. Cellesci ont joué un rôle déterminant dans ma façon d'accueillir la parole, d'entendre et de me situer dans une fonction et une posture analytique qui n'était pas requise au préalable. Je pourrais dire, avec Claudine Blanchard-Laville (2008, p. 162):

\begin{abstract}
[que nous avons ce souci de] prendre soin, [nous avons une] pratique du soin psychique, [...] en proposant des espaces groupaux où puissent être conduites des élaborations psychiques à propos des situations professionnelles racontées par les participants autour d'événements ou de situations concrètes dont ils éprouvent qu'ils les ont mis en difficulté ou en souffrance.
\end{abstract}

Pour moi, il s'agit de prendre particulièrement soin de la parole.

J'ajouterais que ma conception des groupes de parole, aujourd'hui, partage avec l'analyse institutionnelle cette idée de construire un dispositif spatial et temporel dans lequel une parole plus "libre" puisse émerger. Mais si l'assemblée générale se voulait occuper une place centrale dans l'institution, les groupes de parole ont, au contraire, été pensés comme des lieux "à part", en dehors du quotidien et de la routine: des lieux déterritorialisés par rapport aux temps et aux lieux institués de la parole dans l'institution. Par ailleurs la fonction de la parole 
des Sujets et la nature du cadre construit, ainsi que la place des cliniciens, y sont différentes. La clinique institutionnelle opère dans un registre essentiellement imaginaire, là où les groupes de parole d'orientation psychanalytique répondent à d'autres exigences que je situerais à la croisée des résonances psychiques et d'un cadre à forte imprégnation symbolique. Les groupes de parole mettent à l'épreuve ce qu'il en est du désir de l'analyste (LACAN, 2001), c'est-à-dire la position que l'on peut soutenir au regard de la demande qui nous est adressée, du cadre que nous pouvons par conséquent "tenir" pour l'autre, y compris si celui-ci est demandeur d'un tel cadre. C'est bien ce désir et ses ressorts éthiques, qu'il m'a fallu mobiliser pour faire que les groupes de parole avec les adolescents puissent se tenir dans les établissements scolaires, pour qu'ils puissent dépasser les formes d'expression collégienne et lycéenne usuelles, mais surtout pour que nous puissions y accueillir, depuis la joie jusqu'à l'anomie, tout le défilé des problématiques des sujets adolescents.

\section{UN CONTEXTE DE CRISE DE LA RELATION ENTRE LES GÉNÉRATIONS ET LES SEXES}

Lors de notre accueil dans un collège et un lycée qui ont constitué nos principaux terrains de recherche, nous avons été très positivement investis par les équipes éducatives (enseignants et direction). Il leur semblait que notre projet de recherche sur la construction adolescente rencontrait justement ce qui leur était problématique dans leurs établissement scolaires. Il s'y exerçait fortement par contraste avec d'autres milieux sociaux, un mélange idéologique et subjectif très conflictuel fait de domination masculine collective sur les jeunes filles et, simultanément, d'une sorte d'abattement des garçons pris un par un, et, enfin, d'intolérance affichée de certains élèves envers l'homosexualité masculine, mêlée d'agressivité physique et verbale assez permanente entre les sexes. Dans les dires des adultes, un fait sortait de cet ensemble: la violence entre jeunes filles était récurrente, donnant lieu à des échanges de coups, des "embrouilles", des menaces entre jeunes. Régulièrement, les établissements étaient le théâtre de ce que j'ai appelé, en entendant ces adultes, une "flambée" du sexuel: les élèves étaient dits "intenables", "violents", se bagarrant "méchamment". Mais, nous ne réussissions pas à délier dans leurs récits ce qui était propre au pulsionnel adolescent, ce qui relevait des fantasmes individuels ou collectifs des jeunes et des projections des adultes, et ce qui dépendait d'un climat institutionnel et relationnel dégradé.

Des faits avaient "mis le feu au poudre": des petites vidéos pornographiques avaient circulé sur les téléphones mobiles ou sur Internet qui auraient mis en scène des élèves ou des professeurs; il y aurait eu, par ailleurs des réactions très virulentes d'élèves musulmans à des films proposés au cinéclub parce que ces films montraient des relations intimes à l'écran; enfin, les questions sexuelles et relationnelles entre adolescents, entre professeurs, voire entre adolescents et adultes faisaient l'objet de rumeurs incontrôlables participant à la détérioration du climat. 
Par ailleurs, ces mêmes adultes s'interrogeaient face à ce qu'ils ressentaient comme une absence de projets et de capacités à se penser dans le futur chez ces jeunes, un enfermement dans le présent, une difficulté à se projeter s'accompagnant d'une autre: celle de se confier à des adultes.

Voici donc comment notre projet de recherche a été rejoint par les équipes des établissements scolaires qui nous ont fait les destinataires de leurs préoccupations face à des constructions adolescentes les inquiétant. Ils étaient donc presque soulagés que nous proposions des groupes de parole!

\section{L'ORGANiSATION DU DISPOSITIF DES GROUPES DE PAROLE}

Les sept groupes de parole que nous avons menés dans le temps scolaire pendant plusieurs mois ont concerné une cinquantaine de jeunes de 14 à 18 ans (élèves de $4^{\text {ème }}, 3^{\text {ème }} 2^{\text {nde }}$ ). Au collège, les groupes étaient non mixtes et transversaux aux classes, à la demande de la direction. Les parents avaient donné leur autorisation pour que leur enfant participe. Au lycée, les groupes étaient mixtes et la direction avait estimé pouvoir se passer de l'autorisation parentale, les jeunes gens et jeunes filles étant suffisamment responsables pour décider librement de participer ou non au groupe de parole. Les animateurs des groupes étaient toujours deux, plus parfois un étudiant doctorant en formation. Les binômes d'animation ont varié dans leur composition : non mixtes au collège, mixtes ou pas au lycée en fonction des disponibilités. Signalons que ces variations ont eu leur importance dans la situation.

Il s'agissait de créer un espace-temps de parole collective dans les collèges et lycées, un espace bien démarqué des temps d'expression habituels. Il nous paraissait important de proposer un lieu dans lequel ils puissent se parler et parler à des adultes qui ne soient pas les habituelles figures adultes tutélaires. Dans ce cadre groupal, il est évident que se sont actualisés les rapports de sexe et de générations, qui sont au cœur de notre projet de recherche, et dont nous avions aussi compris qu'ils étaient très problématiques pour ces adolescents et pour leurs rapports avec les adultes de la communauté scolaire.

Nous n'avions pas, à l'origine, envisagé de groupes de parole en tant que tels, mais des focus groupes, c'est-à-dire des groupes orientés par les questions des chercheurs et non par la demande des adolescents. Rapidement, pourtant, c'est bien la nécessité d'instituer de tels groupes mettant au centre une parole adolescente et la demande de ces sujets, plutôt que nos questions de chercheurs, qui s'est imposée à nous.

Nous soutenons, aujourd'hui, dans l'après-coup, qu'une parole subjectivée a pu surgir à travers ce dispositif. Cette parole, dans le transfert aux adultes animateurs, a pu se délier parce que nous avions institué un lieu et un temps de parole "libre", en tous cas "à part" bien que dans l'institution, un lieu démarqué des formes d'expression collégiennes et lycéennes usuelles. Le dispositif reposait sur un cadre défini ainsi: la proposition en était relayée par des adultes (équipe de direction au collège, professeurs principaux au lycée), la participa- 
tion était volontaire, nous avons en général fixé un calendrier préalable de 4 séances, tout en laissant ouverte la possibilité de prolongation pour les groupes le souhaitant. Les séances duraient 50 minutes (quelque fois nous avons débordé un peu, emportés par la parole adolescente), elles étaient enregistrées après nous être assurés du consentement des participants.

Nous marquions la spécificité du groupe de parole en structurant une forme de l'échange inhabituelle pour ces jeunes. Nous nous présentions aux adolescents selon la double identité de chercheurs et de cliniciens tout en donnant des explications sur notre projet de recherche, et en les invitant à investir cet espace de parole. Puis nous énoncions des règles fondamentales concernant la durée, la discrétion et la confidentialité de ce qui s'échangeait dans le groupe, le respect de la parole des autres, l'écoute sans jugement autant que possible, mais aussi l'anonymisation de leurs propos dans notre usage pour la recherche. Ce point des règles nous paraissaient d'autant plus important à préciser que les établissements scolaires étaient tous aux prises avec des rumeurs puissantes et par ailleurs ces institutions semblaient avoir une pratique très peu cohérente des règles et des contraintes dans la vie quotidienne ordinaire.

En fait, par ce dispositif qui avait recueilli l'accord des équipes de direction et des jeunes gens, tous volontaires pour participer, nous avons amené la communauté scolaire à reconnaître l'importance d'un espace-temps de socialité, un espace où le lien social se faisait par la parole. Pas n'importe quelle parole: une parole adressée à un groupe fait de pairs et d'adultes “auditeurs", c'est-à-dire entendant ce qui se disait là. Plusieurs jeunes ont témoigné du fait que c'était pour eux la première fois qu'ils rencontraient une telle audition. Sans doute aussi le cadre très contenant que nous procurions à ces groupes avait-il un effet protecteur pour leurs énoncés et pour leurs interactions. Le cadre clinique que nous avons proposé a favorisé l'émergence d'un groupe adolescent moins anomique que ne sont les additions de collégiens ou de lycéens "organisés" comme un ensemble par la seule logique institutionnelle ou instrumentale qui les réifie d'autant plus qu'ils sont des sujets vulnérables dans l'institution.

Nous nous sommes prêtés à ce que ces adolescents puissent éprouver ans l'ici et maintenant du groupe leur parole et leur pensée. J'ai personnellement ressenti dans mon travail que certains adolescents me poussaient dans une posture clinique inspirée par Bion où il me fallait métaboliser et en quelque sorte transformer des parties éparses et assez chaotiques et agressives de leurs énoncés pour les soutenir dans leur parole mais aussi la rendre entendable par les autres. Au-delà de la tenue du cadre clinique, de son soutien malgré quelques attaques à notre autorité sur ce cadre par un ou deux jeunes les plus "paumés", nous étions porteurs aussi d'une éthique de la parole, garants des propos qui s'échangeaient ici, de leur confidentialité, et animés d'un désir de favoriser le lien social. Il s'agissait de permettre l'émergence d'une parole de sujets dans le moment du groupe de parole.

Nous avions aussi pour trame notre désir de leur faire tisser des liens avec leur passé et avec leur devenir, ainsi que d'identifier les processus de leur construction en tant qu'individus sexués, thèmes centraux de notre recherche. 
Nos questions de recherche venaient toucher un point sensible de leurs problématiques de sujets aux prises avec les effets ravageurs de notre époque. Cette opportunité de parler a été saisie et plusieurs groupes ont souhaité prolonger audelà des quatre séances que nous avions initialement fixées. Je pense aussi que le cadre fixé au préalable, en tant que durée relativement brève, a pu produire un effet sur la mise au travail de la parole.

Nous avons eu le sentiment de permettre la construction, au fil des séances, d'un récit à plusieurs voix, un "faire récit" collectif, dans lequel un "nous" parvenait à surgir. Ce fut assez laborieux et parfois même un peu chaotique, la parole pouvait être très effervescente et pas toujours très ordonnée. Mais à certains moments, nous avons aussi pu voir se manifester, chez ces jeunes, une véritable éthique de la discussion, au sens d'Habermas. Cet effet d'une parole "libérée" sur la possible construction d'un récit contrastait soudain avec les problèmes langagiers considérables, la pauvreté du vocabulaire, le champ lexical très restreint de ces jeunes que nous avaient signalés les enseignants. Il faut dire que la jeunesse des quartiers populaires se démarque dans la société par une pratique de la langue alliant le langage vernaculaire des cités, des emprunts au patois des pays d'origine, le tout constituant un "parler jeune" fréquemment peu accessible aux adultes dans son sens et surtout dans son rythme extrêmement rapide et saccadé.

Dans ces groupes de parole, nous avons immédiatement perçu qu'il se passait quelque chose d'inédit car ces sujets nous disaient ne pas avoir l'expérience d'être écoutés et entendus aussi bien dans leur famille qu'à l'école. Notre intérêt en tant que chercheurs fut aussi de constater que dans les groupes un tout autre discours que ceux que nous avions captés durant les observations pouvait s'entendre sur leurs problématiques en tant qu'adolescents, Sujets sexués, mais aussi en tant que fils et filles de parents tenus aux marges de l'institution scolaire et détenteurs d'histoires de migration très frappantes pour leurs enfants.

\section{UNE DÉMARCHE CLINIQUE}

Nous avons pris d'emblée une optique assez différente de ce qu'avait entrepris dans les écoles, il y a une vingtaine d'années, Claire Rueff-Escoubès (1997) en lien avec Gérard Mendel et qui avait consisté à favoriser l'expression lycéenne à l'école pour encourager la socialisation et la démocratie scolaire. Au cœur de leur dispositif était posé un acte de formation des adultes, (conseillers pédagogiques et enseignants) reposant sur l'hypothèse qu'ils étaient le rouage permettant un changement des attitudes des adultes et des adolescents. Pour nous, il s'agissait de poser un cadre clinique dans lequel les adolescents pouvaient venir parler des questions non pas scolaires, mais adolescentes. Nous souhaitions entendre non pas des élèves, mais des sujets, et comprendre comment ils négocient avec et dans l'espace scolaire leurs problématiques adolescentes quant à leur identité sexuée et à leur positionnement générationnel. Nous ne visions pas d'effets particuliers sur la situation scolaire. Et pourtant il s'en est produit car notre dispositif avait la portée d'un lieu instituant en libérant, même si que très modestement, la parole dans les établissements scolaires où elle est généralement très cloisonnée et "spécialisée". 
Là où nous rejoignons l'équipe de Mendel c'est sur sa notion de “cadre du dispositif" car en effet nous avons eu le souci d'instituer un cadre contenant pour cette parole et pour les sujets qui s'exposaient. Un cadre "suffisamment bon" au sens de Winnicott, permettant qu'une parole adolescente se construise entre les adolescents impliqués par la recherche, mais aussi dans notre propre pensée les accompagnant dans ce processus de subjectivation.

Il s'agit en effet d'instaurer non seulement un lieu et un temps de la parole, mais aussi de l'écoute, ce qui n'était pas la moindre des gageures, tant les jeunes auxquels nous avons eu affaire sont loin d'avoir cette capacité dans leurs modalités groupales et individuelles. Il est probable que cette institution de la "parole entendante" dans un groupe fonctionnant avec ses lois propres, énoncées par les cliniciens en début de séance et souvent rappelées, a eu cet effet de cadre, en même temps que nous n'étions pas hors cadre institutionnel: c'est ce dernier qui nous donnait notamment les limites temporelles (scansion et rythme des séances) et les espaces des rencontres dans lequel les séances prenaient place, tout en les signifiant comme des espaces bien distincts.

Au niveau morphologique le plus immédiat, déjà, ces groupes détonnaient car ils ne se coulaient pas dans la forme scolaire mise en évidence par Guy Vincent (1994). Ils en furent même une sorte de subversion rapidement comprise par les adolescents qui ont montré un certain zèle à défaire le dispositif de la classe pour installer l'espace spécifique de la parole du groupe. Selon les situations, les séances commençaient par de rapides et sonores mouvements de tables et de chaises pour "planter le décor" c'est-à-dire former avec le mobilier le cercle de parole, ceci étant notre exigence. Les tables n'étaient pas systématiquement enlevées, nous faisions avec les contraintes des lieux et avec ce que manifestaient les jeunes, qui, la plupart du temps, ont préféré conserver les tables comme une sorte de rempart de protection balisant l'espace intermédiaire entre soi et non soi. Voilà l'image très winnicottienne que cette scène rituelle m'a inspirée. Les tables pouvaient avoir aussi une autre fonction, celle de dissimuler des gestes et des touchers furtifs, comme se tenir par la main, se pincer ou se caresser entre filles. A défaut de table, ce furent souvent les sacs à dos qui étaient tenus serrés contre soi, surtout par les jeunes filles, comme un bouclier devant le ventre et les seins, ou les écharpes que l'on malaxe et tripote à une ou deux filles, côte à côte, dans un geste répétitif; tandis que les jeunes hommes gardaient leurs manteaux ou vestes de jogging, comme s'il s'agissait d'une seconde peau, protégeant une enveloppe corporelle menacée d'intrusion, le groupe et le monde extérieur des adultes que nous présentifions incarnant cette menace imaginaire.

Je dirais que notre souci, dans un registre plus symbolique cette foi-ci, était aussi de constituer un lieu d'adresse: un lieu où une parole adolescente puisse être adressée. Au cœur de notre dispositif à nous, il y avait donc cette question de l'adresse. Question que j'articulerais avec une perspective théorique sans doute plus lacanienne que freudienne en soutenant que le groupe de parole actualisait un rapport de places (adultes/adolescents, hommes/femmes), et un rapport de générations dans lequel se jouait et se répétait dans le transfert pour chaque adolescent concerné, la question symbolique de sa propre place de 
sujet, de ses identifications et de ses problématiques. En disant cela, j'indique que nous avons travaillé la question du transfert et du contre-transfert non pas tant du côté des affects mobilisés dans la relation intersubjective, même si cette question s'est posée fortement à nous, mais plutôt du côté des rapports de places tels qu'ils se manifestaient dans la rencontre adultes/adolescents. Rapports de places que ces jeunes filles et jeunes gens n'ont cessé d'interroger : qui étions-nous pour nous intéresser à eux? Etions-nous du côté de la direction, des professeurs ou de l'Education nationale? A quel Autre, les adolescents s'adressaient-ils, audelà de nous-mêmes? Pourquoi notre équipe était-elle formée de plus âgés ("les professeurs") et de plus jeunes (nos "élèves")? Je rappelle que l'animation des groupes de parole associait autant que possible un enseignant-chercheur et un doctorant en formation clinique. Dans le travail du groupe, du transfert s'est fortement engagé entre les adolescents et les adultes, selon des modalités très variables en fonction de la composition de l'équipe des animateurs: hormis l'âge, les configurations sexuées ont eu une très grande importance. Pourtant, dans ce dispositif des groupes de parole, la question de notre différence générationnelle ne pesait pas de la même façon que dans les autres situations où nous avions été observateurs Sans doute parce que notre présence au groupe, du fait de la posture que nous avions adoptée, n'était pas “adulto-centrée" comme le sont les enseignements. L'empathie que nous avons mise en œuvre pour soutenir la parole des adolescents, n'a pas empêché que les interactions, les échanges soient pris dans un subtil jeu d'adresses et de codes symboliques, un jeu, discret, d'observations et de relations réciproques que notre présence a mis au jour, de manifestations de socialité produites en notre présence, de gestes et de discours échangés devant nous, nous faisant les témoins de leur rapport aux autres (camarades, adultes des équipes éducatives, enseignants) autant qu'à nous mêmes.

A mon initiative deux groupes de parole ont été proposés au collège, à la classe des "durs", celle qui posait le plus problème, classe constituant une sorte de relégation dans la relégation. Les jeunes les plus en décrochage et en difficulté scolaire s'y retrouvaient rassemblés par une sorte de logique institutionnelle brutale. Et en animant ces groupes, nous avons certes entendu ce qui pouvait rendre ces sujets si insupportables à l'institution dans les modalités de leur parole et dans leur attaque au cadre (notre dispositif) mais nous avons aussi pu mesurer toute la fantasmatique dont ces mêmes adolescents sont l'objet dans leur collège, mis en position de "monstres" inquiétants. Ainsi, le chef d'établissement m'a demandé à plusieurs reprises si nous n'avions pas peur qu'on nous laisse seuls avec ces groupes!

\section{L'AUTRE DANS LA RENCONTRE}

Ces jeunes gens, même les plus en difficulté, ne sont évidemment pas assignables aux stéréotypes et réductibles aux représentations qui leur sont attachés déjà par le fait que leurs modalités d'inscription dans les liens sociaux et leurs relations avec leurs pairs et avec les adultes sont multiples. Ce sont des sujets pratiquement tous issus de familles pauvres et populaires, ayant pour la plupart d'entre elles des histoires d'immigration et d'exil, ainsi que des problématiques de précarisation et de disqualification. Ils sont pratiquement tous des fils et des 
filles de parents porteurs de ces récits migratoires qui ont pu ou n'ont pas pu se dire et se transmettre; de parents qui sont eux-mêmes des sujets vulnérables au plan économique et social. Ils habitent les "Quartiers", ils n'ont connu pour beaucoup d'entre eux que ces espaces urbains relégués, faits d'entrelacs de cités et de zones pavillonnaires qu'ils disent aimer.

Une rencontre s'est produite entre nous et eux qui pourrait être mise au compte de ce que Levinas dit à propos de la rencontre du "visage de l'Autre" (LEVINAS, 1982). Car ces adolescentes et adolescents en prenant une consistance, une réalité, nous ont marqués, nous chercheurs et cliniciens, par leurs dires -d'authentiques énonciations- et par leur présence, sans doute aussi par la responsabilité manifestée par quelques uns, qui contrastaient avec tout ce qu'on leur prête dans la société: la violence, l'irrespect et leur propension à développer un sentiment victimaire. L'altérité, selon cette acception, tient à une rencontre sur le vif et au tranchant d'une relation entre "pas semblables", ce que les adolescents nous ont clairement signifié: nous n'étions pas semblables en âge, pas semblables en statut, pas semblables du point de vue de nos places respectives. Je garde à l'esprit cette phrase d'un jeune homme qui nous accueillait régulièrement dans sa classe par un interrogatoire sur nos origines et un jour produisit cette interprétation radicale: "Allez... vous venez de l'Inspection ! car pourquoi sinon vous vous intéressez à nous qui sommes des moins que rien, pour tout le monde?". Nous étions étranges et étrangers les uns aux autres, mais ils étaient au moins autant curieux de nous, que nous l'étions d'eux! Ils cherchaient aussi à "accrocher" sur notre équipe quelque chose d'eux, de connu d'eux. Ainsi, à maintes reprises leurs questions fusèrent à propos des liens existant entre les universitaires de notre équipe et les étudiants doctorants en train d'apprendre à nos côtés, ou encore à propos du fait que plusieurs étudiants étaient "des étrangers" (Italienne, Colombienne, Sénégalaise). Nous étions semble-t-il différents des adultes qu'ils ont l'habitude de rencontrer parce les liens intergénérationnels que nous donnions à voir entre nous leur étaient énigmatiques. Ils ne manquèrent pas de les interroger de diverses manières. Sans doute cette altérité là a-t-elle permis que s'organise du transfert à notre endroit. Les groupes de parole nous ont ainsi permis de mettre au jour la "négociation", l'ajustement permanent que ces jeunes doivent opérer au quotidien lorsqu'ils sont dans l'espace social qu'est l'école.

Pour conclure, je dirais que beaucoup d'autres questionnements peuplent notre travail. Nous n'en sommes qu'aux débuts de notre élaboration. En tous cas la forme groupe de parole par sa dimension instituante a rencontré quelque chose des subjectivités adolescentes en ce qu'elles viennent interroger l'ordre institué et disciplinaire de l'école et les relations assujettissantes qu'il produit. La rupture que nous avons créée avec un certain ordre des choses dans l'institution scolaire a semble-t-il permis aux adolescents d'entendre dans ce geste et cet acte des groupes de parole qu'ils les affranchissaient d'un institué empêchant. C'est sans doute là que se situe la condition de possibilité pour qu'une parole plus pleine puisse prendre place dans l'institution. 


\section{Notes}

${ }^{1}$ Agence Nationale de la Recherche, “Copsy-enfant”, 2005-2008.

\section{RÉFÉRENCES}

BLANCHARD-LAVILLE, $\mathrm{C} . \mathrm{Du}$ soin psychique aux enseignants: psychopathologie du quotidien de l'enseignant. Cliniques méditerranéennes, [S.1.], n. 77, p.159-176, 2008.

CASTEL, R.; HAROCHE, C. Propriété privée, propriété sociale, propriété de soi: entretiens sur la construction de l'individu moderne. Paris: Fayard, 2001.

CASTORIADIS, C. L'institution imaginaire de la société. Paris: Seuil, 1975.

LACAN, J. Le séminaire: le transfert. Paris: Seuil, 2001. Livre VIII.

LEVINAS, E. Ethique et infini: dialogues avec Philippe Nemo. Fayard: France Culture, 1982.

LOURAU, R. Instituant contre institué. Paris: Anthropos, 1969.

LOURAU, R. L'analyse institutionnelle. Paris: Minuit, 1970.

ROUCHY, J-C. La conception du dispositif de groupe dans différents cadres institutionnels. Revue psychanalytique de groupe, [S.1.], n. 47, p. 9-23, 2006.

RUEFF-ESCOUBÈS, C. La démocratie dans l'école: une pratique d'expression des élèves. Paris: Syros, 1997. Préface de Gérard Mendel.

VINCENT, G. L'éducation prisonnière de la forme scolaire. Lyon: PUL, 1994.

Reçu: Juin 2009

Accepté: Novembre 2009 